\title{
A remark on Secelean-Wardowski's fixed point theorems
}

\author{
Satit Saejung ${ }^{1,2}$ and Pinya Ardsalee ${ }^{3 *}$ (ID
}

${ }^{\text {"Correspondence: }}$

ardsalee.p@msu.ac.th

${ }^{3}$ Department of Mathematics,

Mahasarakham University,

Mahasarakham, Thailand

Full list of author information is

available at the end of the article

\begin{abstract}
In this paper we give a simple proof of three fixed point theorems of Secelean and Wardowski by using the fixed point result of Jachymski et al. Our result is established with weaker assumptions than the three theorems. Furthermore, the recent result of Secelean et al. in the setting of a complete metric space can be also deduced by our theorem.
\end{abstract}

Keywords: $\psi$ F-contraction; Semimetric space; Fixed point

\section{Introduction}

Banach's contraction principle is one of the famous fixed point theorems which play a key role in various branches in mathematical models. Many mathematicians have attempted to generalize this result in many ways.

The fixed point theorem of Jachymski et al. [1] and that of Wardowski [5] were proposed in 1995 and in 2012, respectively. Both of them are generalizations of Banach's contraction principle. In 2016, Secelean and Wardowski [4] established three fixed point theorems (see Theorems SW1-SW3) which are the generalizations of Wardowski's fixed point theorem (see Theorem W).

In this paper, our purpose is to show that the results of Secelean and Wardowski can be easily deduced from those of Jachymski et al. We discuss that some conditions of their results can be omitted. Finally, we show that in the setting of complete metric spaces the recent result of Secelean et al. [3] proposed in 2019 can be deduced from our theorem.

We now recall the following three theorems which are known as fixed point theorems of Banach, of Jachymski et al., and of Wardowski, respectively. The definitions of the relevant terms are given in Sect. 2.

Theorem B Suppose that $(X, d)$ is a complete metric space and $\gamma \in(0,1)$. Suppose that a mapping $T: X \rightarrow X$ satisfies

$$
d(T x, T y) \leq \gamma d(x, y) \quad \text { for all } x, y \in X
$$

Then $T$ has a unique fixed point, that is, there exists a unique element $p \in X$ such that $p=T p$. Moreover, if $x \in X$, then $\lim _{n} d\left(T^{n} x, p\right)=0$.

(c) The Author(s) 2022. This article is licensed under a Creative Commons Attribution 4.0 International License, which permits use, sharing, adaptation, distribution and reproduction in any medium or format, as long as you give appropriate credit to the original author(s) and the source, provide a link to the Creative Commons licence, and indicate if changes were made. The images or other third party material in this article are included in the article's Creative Commons licence, unless indicated otherwise in a credit line to the material. If material is not included in the article's Creative Commons licence and your intended use is not permitted by statutory regulation or exceeds the permitted use, you will need to obtain permission directly from the copyright holder. To view a copy of this licence, visit http://creativecommons.org/licenses/by/4.0/. 
Theorem JMS Suppose that a semimetric space $(X, D)$ is Hausdorff and complete. Suppose that $\varphi:[0, \infty) \rightarrow[0, \infty)$ is nondecreasing and $\lim _{n} \varphi^{n}(t)=0$ for all $t>0$. Suppose that $T:$ $X \rightarrow X$ satisfies the following condition:

$$
D(T x, T y) \leq \varphi(D(x, y)) \quad \text { for all } x, y \in X
$$

Suppose that one of the three conditions in Proposition 5 holds (see Sect. 2). Then $T$ has a unique fixed point $p \in X$ and $\lim _{n} D\left(T^{n} x, p\right)=0$ for all $x \in X$.

The following theorem of Wardowski is slightly modified to fit the presentation of the more general setting of its generalization proposed by Secelean and Wardowski [4]. Let us recall some notations. For a given metric space $(X, d)$, let $\operatorname{diam} X:=\sup _{x, y \in X} d(x, y)$. For each $v, \lambda \in(0, \infty]$, the notation " $v>\lambda$ " means $v>\lambda$ if $\lambda<\infty$ and $v=\infty$ if $\lambda=\infty$.

Theorem W Suppose that $(X, d)$ is a complete metric space and $v>\operatorname{diam} X$. Suppose that $F:(0, v) \rightarrow(-\infty, \infty)$ satisfies the following conditions:

(A1) For each sequence $\left\{\alpha_{n}\right\} \subset(0, v)$, it follows that

$$
\lim _{n} \alpha_{n}=0 \Leftrightarrow \lim _{n} F\left(\alpha_{n}\right)=-\infty
$$

(A2) $F$ is increasing, that is, $0<\alpha<\beta<v \Rightarrow F(\alpha)<F(\beta)$;

(A3) There exists a real number $\lambda \in(0,1)$ such that $\lim _{\alpha \rightarrow 0^{+}} \alpha^{\lambda} F(\alpha)=0$.

Suppose that $\tau>0$ and $T: X \rightarrow X$ satisfy

$$
d(T x, T y)>0 \quad \Rightarrow \quad \tau+F(d(T x, T y)) \leq F(d(x, y)) .
$$

Then $T$ has a unique fixed point $p \in X$ and $\lim _{n} d\left(T^{n} x, p\right)=0$ for all $x \in X$.

Theorem B is just a special case of Theorem JMS where $\varphi(t):=\alpha t$. Note that every complete metric space $(X, d)$ is a semimetric space which is Hausdorff and complete and satisfies Condition (C3). In fact, the triangle inequality implies Condition (C3) (see Proposition 5 in Sect. 2).

Theorem B is also a special case of Theorem W. In fact, if $d(T x, T y) \leq \gamma d(x, y)$ for all $x, y \in X$, then $\tau+F(d(T x, T y)) \leq F(d(x, y))$ for all $x, y \in X$ with $T x \neq T y$ where $F(\alpha):=\ln \alpha$ and $\tau:=-\ln \gamma$.

Let us recall three fixed point theorems of Secelean and Wardowski [4].

Theorem SW1 Suppose that $(X, d)$ is a complete metric space and $v>\operatorname{diam} X$. Suppose that $F:(0, v) \rightarrow(-\infty, \infty)$ satisfies conditions $(A 1)$ and

(A2') $\sup _{t \in M} F(t)<\infty$ for every bounded set $M \subset(0, v)$.

Let $\mu>\sup F:=\sup _{0<t<v} F(t)$. Suppose that $T: X \rightarrow X$ and $\psi:(-\infty, \mu) \rightarrow(-\infty, \mu)$ satisfy the following conditions:

(B1) $F(d(T x, T y)) \leq \psi(F(d(x, y)))$ for each $x, y \in X$ with $T x \neq T y$;

(B2) $\psi$ is increasing and $\lim _{n} \psi^{n}(t)=-\infty$ for all $t \in(-\infty, \mu)$;

(B3) There exists $x_{0} \in X$ such that the sequence $\left\{T^{n} x_{0}\right\}$ is bounded.

Then $T$ has a unique fixed point $p \in X$ and $\lim _{n} d\left(T^{n} x, p\right)=0$ for all $x \in X$. 
Theorem SW2 Suppose that $(X, d)$ is a complete metric space and $v>\operatorname{diam} X$. Suppose that $F:(0, v) \rightarrow(-\infty, \infty)$ satisfies Conditions (A1), (A2'), and (A3). Let $\mu>\sup F$. Suppose that $T: X \rightarrow X$ and $\psi:(-\infty, \mu) \rightarrow(-\infty, \mu)$ satisfy Conditions $(B 1),(B 2)$, and

(B4) there exist $x_{0} \in X$ and an integer $N$ such that $x_{0} \neq T x_{0}, \psi^{N}\left(F\left(d\left(x_{0}, T x_{0}\right)\right)\right)<0$, and $\sum_{k \geq N}\left|\psi^{k}\left(F\left(d\left(x_{0}, T x_{0}\right)\right)\right)\right|^{-1 / \lambda}<\infty$ and $\lambda$ is given from Condition (A3).

Then $T$ has a unique fixed point $p \in X$ and $\lim _{n} d\left(T^{n} x, p\right)=0$ for all $x \in X$.

Theorem SW3 Suppose that $(X, d)$ is a complete metric space and $v>\operatorname{diam} X$. Suppose that $F:(0, v) \rightarrow(-\infty, \infty)$ satisfies Condition $(A 1)$. Let $\mu>\sup F$. Suppose that $T: X \rightarrow X$ and $\psi:(-\infty, \mu) \rightarrow(-\infty, \mu)$ satisfy Conditions (B1), (B2), and

(B5) $\psi$ is continuous and the set of discontinuities of $F$ is at most countable.

Then $T$ has a unique fixed point $p \in X$ and $\lim _{n} d\left(T^{n} x, p\right)=0$ for all $x \in X$.

Remark 1 Theorem SW1 improves Theorem W in the following ways:

- Condition (A3) in Theorem W is not required in Theorem SW1;

- Condition (A2) in Theorem W implies Condition (A2') in Theorem SW1;

- If $\psi(t):=t-\tau$ for all $t \in(-\infty, \infty)$, then Condition (B2) is satisfied.

\section{Definitions and preliminaries}

Definition 2 Suppose that $X$ is a nonempty set and $D: X \times X \rightarrow[0, \infty)$. We say that $(X, D)$ is a semimetric space if the following conditions are satisfied:

(1) $D(x, y)=0 \Longleftrightarrow x=y$;

(2) $D(x, y)=D(y, x)$ for all $x, y \in X$.

If a semimetric space $(X, D)$ satisfies the triangle inequality, that is, $D(x, y) \leq D(x, z)+D(z, y)$ for all $x, y, z \in X$, then we say that $(X, D)$ is a metric space.

Definition 3 Suppose that $\left\{x_{n}\right\}$ is a sequence in a semimetric space $(X, D)$. We say that

- $\left\{x_{n}\right\}$ converges to $x \in X$ if $\lim _{n} D\left(x_{n}, x\right)=0$;

- $\left\{x_{n}\right\}$ is Cauchy if $\lim _{n} \sup \left\{D\left(x_{l}, x_{m}\right): l>m \geq n\right\}=0$.

Definition 4 We say that a semimetric space $(X, D)$ is

- Hausdorff if $x=y$ whenever $\lim _{n} D\left(x_{n}, x\right)=\lim _{n} D\left(x_{n}, y\right)=0$;

- complete if whenever $\left\{x_{n}\right\}$ is a Cauchy sequence in $X$ it follows that $\left\{x_{n}\right\}$ converges to some element $x \in X$.

It is clear from the definition that every metric space is Hausdorff. The concept of semimetric spaces requires only few assumptions. It is natural to impose some further condition on the space. The following result was proposed by Jachymski et al. [1].

Proposition 5 ([1]) Suppose that $(X, D)$ is a semimetric space. Then the following statements are equivalent.

(C1) There exists a real number $r>0$ such that

$$
\sup \{\beta(x ; r): x \in X\}<\infty,
$$

where $\beta(x ; r):=\sup \{D(z, y): D(x, z)<r$ and $D(x, y)<r\}$. 
(C2) There are two real numbers $\delta, \eta>0$ such that $D(x, y) \leq \eta$ whenever $x, y, z \in X$ with $D(x, z)+D(z, y)<\delta$.

(C3) For all sequences $\left\{x_{n}\right\},\left\{y_{n}\right\}$, and $\left\{z_{n}\right\}$ in $X$, the following implication holds:

$$
\lim _{n} D\left(x_{n}, z_{n}\right)=\lim _{n} D\left(z_{n}, y_{n}\right)=0 \quad \Longrightarrow \quad \lim _{n} D\left(x_{n}, y_{n}\right) \neq \infty
$$

\section{Main results}

In this paper, we show that Theorem SW1, Theorem SW2, and Theorem SW3 are not only a consequence of Theorem JMS but they are also established with weaker assumptions (see Remark 7).

Theorem 6 Suppose that $(X, d)$ is a complete metric space and $v>\operatorname{diam} X$. Suppose that $F:(0, v) \rightarrow(-\infty, \infty)$ satisfies Condition (A1). Let $\mu>\sup F$. Suppose that $T: X \rightarrow X$ and $\psi:(-\infty, \mu) \rightarrow(-\infty, \mu)$ satisfy Conditions $(B 1)$ and (B2). Then T has a unique fixed point $p \in X$ and $\lim _{n} d\left(T^{n} x, p\right)=0$ for all $x \in X$.

Remark 7 Our result improves the three theorems of Secelean and Wardowski in the following ways.

(a) Conditions (A2') and (B3) are not required as was the case in Theorem SW1.

(b) Conditions (A2'), (A3), and (B4) are not required as was the case in Theorem SW2.

(c) Condition (B5) is not required as was the case in Theorem SW3.

We start with the following easy lemma.

Lemma 8 Let $v \in(0, \infty]$. Suppose that $F:(0, v) \rightarrow(-\infty, \infty)$ satisfies Condition (A1). Suppose that $\left\{\alpha_{n}\right\}$ is a sequence in $(0, v)$. Then

$$
\lim _{n} e^{F\left(\alpha_{n}\right)}=0 \Longleftrightarrow \lim _{n} \alpha_{n}=0 .
$$

Lemma 9 Suppose that $(X, d)$ is a metric space. Let $v>\operatorname{diam} X$. Suppose that $F:(0, v) \rightarrow$ $(-\infty, \infty)$ satisfies Condition (A1). Define $D: X \times X \rightarrow[0, \infty)$ by

$$
D(x, y):= \begin{cases}e^{F(d(x, y))} & \text { if } x \neq y \\ 0 & \text { if } x=y\end{cases}
$$

Then the following statements hold:

(1) $(X, D)$ is a Hausdorff semimetric space satisfying Condition (C3).

(2) If $(X, d)$ is complete, then $(X, D)$ is complete.

Proof It is clear that $(X, D)$ is a semimetric space.

(1) We show that $(X, D)$ is Hausdorff. To see this, let $\left\{x_{n}\right\}$ be a sequence in $X$ and $x, y \in X$ such that $\lim _{n} D\left(x_{n}, x\right)=\lim _{n} D\left(x_{n}, y\right)=0$. By Lemma 8 , we have $\lim _{n} d\left(x_{n}, x\right)=$ $\lim _{n} d\left(x_{n}, y\right)=0$. It follows from the triangle inequality of $d$ that $x=y$. To see that $(X, D)$ satisfies Condition (C3), let $\left\{x_{n}\right\},\left\{y_{n}\right\}$, and $\left\{z_{n}\right\}$ be sequences in $X$ such that $\lim _{n} D\left(x_{n}, z_{n}\right)=$ $\lim _{n} D\left(z_{n}, y_{n}\right)=0$. It follows from Lemma 8 that $\lim _{n} d\left(x_{n}, z_{n}\right)=\lim _{n} d\left(z_{n}, y_{n}\right)=0$. This implies that $\lim _{n} d\left(x_{n}, y_{n}\right)=0$ and hence $\lim _{n} D\left(x_{n}, y_{n}\right)=0 \neq \infty$. 
(2) Suppose that $(X, d)$ is complete. To see that $(X, D)$ is complete, let $\left\{x_{n}\right\}$ be a Cauchy sequence in the semimetric space $(X, D)$, that is,

$$
\limsup _{n}\left\{D\left(x_{l}, x_{m}\right): l>m \geq n\right\}=0
$$

We show that $\lim _{n} \gamma_{n}=0$ where $\gamma_{n}:=\sup \left\{d\left(x_{l}, x_{m}\right): l>m \geq n\right\}$. Note that $\gamma_{n+1} \leq \gamma_{n}$ for all $n \geq 1$. Suppose $\lim _{n} \gamma_{n} \neq 0$. Then there exists $\varepsilon_{0}>0$ such that $\gamma_{n}>\varepsilon_{0}$ for all $n \geq 1$. In particular, for each $n \geq 1$, there exist two integers $l(n)>m(n) \geq n$ such that $d\left(x_{l(n)}, x_{m(n)}\right)>$ $\varepsilon_{0}$ which implies that $\lim _{n} d\left(x_{l(n)}, x_{m(n)}\right) \neq 0$. It follows from Lemma 8 that

$$
\lim _{n} D\left(x_{l(n)}, x_{m(n)}\right) \neq 0
$$

which is a contradiction. Therefore, $\lim _{n} \gamma_{n}=0$, that is, the sequence $\left\{x_{n}\right\}$ is Cauchy in $(X, d)$. Since $(X, d)$ is complete, there is an element $x \in X$ such that $\lim _{n} d\left(x_{n}, x\right)=0$ and hence $\lim _{n} D\left(x_{n}, x\right)=0$. This completes the proof.

Lemma 10 Let $\mu \in(0, \infty]$. Suppose that $\psi:(-\infty, \mu) \rightarrow(-\infty, \mu)$ is increasing and $\lim _{n} \psi^{n}(t)=-\infty$ for all $t \in(-\infty, \mu)$. Define $\varphi:[0, \mu) \rightarrow[0, \mu)$ by

$$
\varphi(t):= \begin{cases}0 & \text { if } t=0 \\ e^{\psi(\ln t)} & \text { if } t \in(0, \mu)\end{cases}
$$

Then $\varphi$ is nondecreasing and $\lim _{n} \varphi^{n}(t)=0$ for all $t \in(0, \mu)$.

Proof It is clear that $\varphi$ is increasing. Let $t \in(0, \mu)$. It is easy to see that $\varphi^{n}(t)=e^{\psi^{n}(\ln t)}$ for all $n \geq 1$. In particular, since $\lim _{n} \psi^{n}(\ln t)=-\infty$, we have $\lim _{n} \varphi^{n}(t)=0$.

We are now ready to prove our main result.

Proof of Theorem 6 Suppose that $X, d, F, T$, and $\psi$ are as the prerequisite of our Theorem 6. We define $D: X \times X \rightarrow[0, \infty)$ and $\varphi:[0, \mu) \rightarrow[0, \mu)$ as in our Lemma 9 and Lemma 10 , respectively. In particular, $(X, D)$ is a semimetric space which is Hausdorff and complete and satisfies Condition (C3). We now prove that

$$
D(T x, T y) \leq \varphi(D(x, y)) \quad \text { for all } x, y \in X
$$

The inequality holds trivially if $T x=T y$. Now, suppose that $x, y \in X$ with $d(T x, T y)>0$. This implies that

$$
F(d(T x, T y)) \leq \psi(F(d(x, y)))
$$

Hence

$$
D(T x, T y)=e^{F(d(T x, T y))} \leq e^{\psi(F(d(x, y)))}=e^{\psi(\ln (D(x, y)))}=\varphi(D(x, y)) .
$$

By Theorem JMS, the mapping $T$ has a unique fixed point $p \in X$ and $\lim _{n} D\left(T^{n} x, p\right)=0$ for all $x \in X$. In particular, $\lim _{n} d\left(T^{n} x, p\right)=0$ for all $x \in X$. This completes the proof. 
In 2019, Secelean et al. [3] established some fixed point theorems which generalize Theorem $\mathrm{W}$ by considering it in quasi-metric spaces and the function $F$ satisfying only Condition (W1). The notion of quasi-metric spaces is more general than that of metric spaces. However, in the setting of metric spaces, we can show that some result in [3] can deduced by our Theorem 6 . Let us recall their result in the setting of complete metric spaces.

Theorem SMW Suppose that $(X, d)$ is a complete metric space. Suppose that $F:(0, \infty) \rightarrow$ $(-\infty, \infty)$ satisfies Condition (A2). Let $\mu>\sup F$. Suppose that $T: X \rightarrow X$ and $\psi$ : $(-\infty, \mu) \rightarrow(-\infty, \mu)$ satisfy Conditions $(B 1)$ and

(B2') $\psi$ is continuous and increasing and $\psi(t)<t$ for all $t \in(-\infty, \mu)$.

Then $T$ has a unique fixed point $p \in X$ and $\lim _{n} d\left(T^{n} x, p\right)=0$ for all $x \in X$.

To deduce Theorem SMW from our result, we need the following two lemmas.

Lemma $11([2])$ Let $F:(0, \infty) \rightarrow(-\infty, \infty)$ be an increasing function and $\left\{t_{n}\right\}$ be a sequence in $(0, \infty)$. Then the following hold:

(a) If $\lim _{n} F\left(t_{n}\right)=-\infty$, then $\lim _{n} t_{n}=0$;

(b) If $\inf F=-\infty$ and $\lim _{n} t_{n}=0$, then $\lim _{n} F\left(t_{n}\right)=-\infty$.

Lemma $12([3])$ Let $\mu>\sup F$. Suppose that $\psi:(-\infty, \mu) \rightarrow(-\infty, \mu)$ is continuous, increasing, and $\psi(t)<t$ for all $t \in(-\infty, \mu)$. Then $\lim _{n} \psi^{n}(t)=-\infty$ for all $t \in(-\infty, \mu)$.

A proof of Theorem SMW via our Theorem 6 where $v=\infty$ We consider the following two cases.

Case 1: For each $x_{0} \in X$, there exists a nonnegative integer $n$ such that $T^{n+1} x_{0}=T^{n} x_{0}$.

This implies that $p:=T^{n} x_{0}$ is a fixed point of $T$. Note that $T$ cannot have more than one fixed point. The conclusion follows.

Case 2: There exists $x_{0} \in X$ such that $T^{n+1} x_{0} \neq T^{n} x_{0}$ for all $n \geq 0$. This implies that

$$
\begin{aligned}
F\left(d\left(T^{n+2} x_{0}, T^{n+1} x_{0}\right)\right) & \leq \psi\left(F\left(d\left(T^{n+1} x_{0}, T^{n} x_{0}\right)\right)\right) \\
& \leq \psi^{2}\left(F\left(d\left(T^{n} x_{0}, T^{n-1} x_{0}\right)\right)\right) \\
& \vdots \\
& \leq \psi^{n+1}\left(F\left(d\left(T x_{0}, x_{0}\right)\right)\right) .
\end{aligned}
$$

It follows from Lemma 12 that $\lim _{n} \psi^{n}\left(F\left(d\left(T x_{0}, x_{0}\right)\right)\right)=-\infty$, and hence $\lim _{n} F\left(d\left(T^{n+2} x_{0}\right.\right.$, $\left.\left.T^{n+1} x_{0}\right)\right)=-\infty$. In particular, inf $F=-\infty$. It follows from Lemma 11 that $F$ satisfies Condition (A1). Note that $\psi$ satisfies Condition (B2) by Lemma 12. Hence the conclusion follows from our Theorem 6.

Acknowledgements

We would like to thank all reviewers for their comments and suggestions which enhanced the presentation of the paper.

Funding

SS was supported by the Thailand Research Fund and Khon Kaen University under grant RSA6280002 and PA was supported by Mahasarakham University. 


\section{Declarations}

Competing interests

The authors declare that they have no competing interests.

Authors' contributions

SS and PA contributed equally and significantly in writing this article. All authors read and approved the final manuscript.

\section{Author details}

${ }^{1}$ Department of Mathematics, Khon Kaen University, Khon Kaen, Thailand. ${ }^{2}$ Research Center for Environmental and Hazardous Substance Management (EHSM), Khon Kaen University, Khon Kaen, Thailand. ${ }^{3}$ Department of Mathematics, Mahasarakham University, Mahasarakham, Thailand.

\section{Publisher's Note}

Springer Nature remains neutral with regard to jurisdictional claims in published maps and institutional affiliations.

Received: 9 December 2020 Accepted: 31 January 2022 Published online: 21 February 2022

\section{References}

1. Jachymski, J., Matkowski, J., Swiatkowski, T.: Nonlinear contractions on semimetric spaces. J. Appl. Anal. 1(2), 125-134 (1995)

2. Secelean, N.A.: Iterated function systems consisting of F-contractions. Fixed Point Theory Appl. 2013, Article ID 277 (2013)

3. Secelean, N.A., Mathew, S., Wardowski, D.: New fixed point results in quasi-metric spaces and applications in fractals theory. Adv. Differ. Equ. 2019, Article ID 177 (2019). https://doi.org/10.1186/s13662-019-2119-z

4. Secelean, N.A., Wardowski, D.: $\psi$ F-contractions: not necessarily nonexpansive Picard operators. Results Math. 70 , 415-431 (2016)

5. Wardowski, D.: Fixed points of a new type of contractive mappings in complete metric spaces. Fixed Point Theory Appl. 2012, Article ID 94 (2012). https://doi.org/10.1186/1687-1812-2012-94

\section{Submit your manuscript to a SpringerOpen ${ }^{\circ}$ journal and benefit from:}

- Convenient online submission

- Rigorous peer review

- Open access: articles freely available online

- High visibility within the field

- Retaining the copyright to your article

Submit your next manuscript at $\gg$ springeropen.com 